\title{
CARACTERÍSTICAS DE LA PLANIFICACIÓN DE LA ESCRITURA EN ESTUDIANTES CON SÍNDROME DE DOWN
}

CHARACTERISTICS OF THE WRITING PLANNING OF SYNDROME DOWN STUDENTS

\author{
Volumen 16, Número 1 \\ Enero - Abril \\ pp. 1-29
}

José Luis Gallego Ortega Antonio Rodríguez Fuentes

Revista indizada en REDALYC, $\underline{\text { SCIELO }}$

Revista distribuida en las bases de datos:

LATINDEX, DOAJ, E-REVIST@S, IRESIE, CLASE, DIALNET, SHERPA/ROMEO, QUALIS,

Revista registrada en los directorios:

ULRICH'S, REDIE, RINACE, OEI, MAESTROTECA, PREAL, CLACSO 


\title{
CARACTERÍSTICAS DE LA PLANIFICACIÓN DE LA ESCRITURA EN ESTUDIANTES CON SÍNDROME DE DOWN \\ CHARACTERISTICS OF THE WRITING PLANNING OF SYNDROME DOWN STUDENTS
}

\author{
José Luis Gallego Ortega ${ }^{1}$ \\ Antonio Rodríguez Fuentes ${ }^{2}$
}

Resumen: La escritura es una habilidad cognitiva compleja de extraordinaria importancia para el desarrollo personal y social de las personas. De ahí que el objetivo de esta investigación fuera conocer cómo planifican la expresión escrita estudiantes con Síndrome de Down que asisten a centros de educación especial en Granada y Jaén (España). Se trata de una investigación de carácter cualitativo fundamentalmente, concebida como un "estudio de caso colectivo" en el que participaron veinticinco chicos y chicas con Síndrome de Down cuyas edades estaban comprendidas entre los 16 y los 21 años. Se utilizó la "entrevista semiestructurada" para obtener los datos y el "análisis de contenido" para interpretarlos. Los resultados obtenidos han revelado cuáles son las estrategias u operaciones de la planificación de la escritura que los y las estudiantes con Síndrome de Down activan durante la elaboración de un texto así como las dificultades más relevantes que encuentran en dicho proceso. Por último, se ofrecen algunas orientaciones didácticas para la enseñanza de la planificación de la escritura.

Palabras clave: PLANIFICACIÓN ESCRITORA, ESCRITURA, SÍNDROME DE DOWN, DIFICULTADES ESCRITORAS, ESTUDIO DE CASOS.

Abstract: The writing is a complex cognitive skill of extraordinary importance for the personal and social development of the people. Therefore the objective of this research is to know how the syndrome down students who attend a special education school in Granada and Jaén (Spain) plan the writing expression. This qualitative research entails a "collective case study" of twenty five syndrome down students of between 16 to 21 years old. The "semi-organized interview" is used to gather data and the "content analysis" is used to interpret them. The results obtained have showed what the strategies and operations of the planning of the writing which are activated by the syndrome down students while the preparation of a text are as well as what the most important difficulties that they find during this process are. Finally, some educational aspects are offered for the teaching in planning the writing.

Key Words: WRITING PLANNING, WRITING, DOWN SYNDROME, WRITING DIFFICULTIES, CASE STUDY.

\footnotetext{
1 Profesor Titular de la Facultad de Ciencias de la Educación de la Universidad de Granada (España). Doctor en Ciencias de la Educación por la Universidad de Granada. Dirección electrónica: jlgalleg@ugr.es

2 Profesor Titular de la Facultad de Ciencias de la Educación de la Universidad de Granada (España). Doctor en Ciencias de la Educación por la Universidad de Granada. Dirección electrónica: arfuente@ugr.es
}

Artículo recibido: 23 de marzo, 2015

Enviado a corrección: 17 de junio, 2015

Aprobado: 5 de octubre, 2015 


\section{Introducción}

La escritura constituye un reto para la investigación, dado que su aprendizaje es fundamental para el desarrollo personal y social de los individuos. La lengua escrita es hoy foco de atención preferente, por razones diversas, entre investigadoras e investigadores de múltiples disciplinas y docentes de todos los niveles educativos. Lectura y escritura son productos del desarrollo cultural y su adquisición y dominio demandan un ambiente alfabetizador que propicie y oriente estos aprendizajes en los que participan procesos psicológicos diferenciados (Canales, Velarde, Meléndez y Lingán, 2013). Sin embargo, lectura y escritura no han concitado el mismo interés investigativo, tal vez porque escribir es más exigente que leer (Alegría y Carrillo, 2014). Mientras que la lectura ha acaparado la atención de la investigación, la escritura ha estado en cierto modo relegada y solo en las últimas décadas se ha incrementado su interés visiblemente, como lo demuestra el número de publicaciones en uno y otro campo. A este efecto, pueden ser ilustrativas las siguientes palabras:

Aunque el aprendizaje de la lengua escrita abarca tanto el aprendizaje de la lectura como el de la escritura, sólo recientemente se ha incorporado el conocimiento de la escritura como elemento explicativo de las diferencias individuales en el aprendizaje de la lengua escrita. (Tolchinsky, Ribera y García-Parejo, 2012, p. 4)

En este sentido, la investigación se ha encargado de desvelar las dificultades escritoras de diferentes grupos de estudiantes y también cuáles son sus potenciales habilidades. Se sabe, por ejemplo, que entre el alumnado de educación primaria y secundaria ocupan un lugar destacado las dificultades relacionadas con la progresión temática, el conocimiento semántico, morfosintáctico y metacognitivo (González y Martín, 2006; Velarde y Canales, 2014), el desconocimiento de las fuentes para generar ideas, la inadecuación del texto a las necesidades de la audiencia o desmerecer la relevancia del uso de borradores (Gallego, 2008b), así como los fallos en el manejo de estrategias cognitivas y metacognitivas (Lecuona, Rodríguez y Sánchez, 2003; Velarde y Canales, 2014) o en el proceso de revisión textual (Salvador y García, 2009). Asimismo, otras investigaciones atribuyen los inconvenientes escritores a ciertos usos inadecuados de las estructuras textuales y de estilo (Ramos, Cuadrado e Iglesias, 2005), a desventajas sociales del alumnado (García, 2009; García y Salvador, 2010), a ciertas discapacidades (Gutiérrez y Salvador, 2006; Rodríguez, 2007; 
Gallego y Rodríguez, 2011) o incluso, a condiciones de sobredotación intelectual (Gallego y González, 2008).

Por otra parte, los estudios realizados con estudiantes que presentan dificultades de aprendizaje revelan que este alumnado lucha contra casi todos los aspectos de la escritura (Re, Pedron y Cornoldi, 2007; Aponte-Henao y Zapata-Zabala, 2013). Efectivamente, tienden a crear composiciones más cortas, menos elaboradas y con más errores mecánicos (Gleason e Isaacson, 2001); carecen del conocimiento de la estructura textual (Newcomer y Barenbaum, 1991) y de las habilidades para la ejecución de los procesos de escritura (Graham, 2006); suelen generar el contenido de forma lineal (Bereiter y Scardamalia, 1987; McCutchen, 1995) e incompleta (Englert y Raphael, 1988) y expresan las ideas sobre el tema sin demasiada relación entre ellas (Williams, 2003) y de forma confusa e incoherente (Troia, 2006). También, dentro de este colectivo, una investigación reveló que los estudiantes con discapacidad intelectual de origen desconocido - aunque suelen consultar diferentes fuentes cuando escriben, estar guiados por motivaciones muy diversas y atender a sus destinatariosmuestran importantes limitaciones escritoras, por ejemplo, desconocer las estructuras textuales, ignorar las ideas o palabras que mayor relación tienen con el tema de escritura 0 infravalorar la importancia de los borradores previos (Gallego, 2008a).

Asimismo, principalmente en las últimas décadas, se han publicado estudios en los que se pone de manifiesto cuáles son las potencialidades escritoras (p. ej., la riqueza léxica o tener muy en cuenta a la audiencia) y qué inconvenientes relevantes muestran (p. ej., la búsqueda de nuevas fuentes de información o el manejo de recursos mnemónicos para elaborar un texto) los estudiantes universitarios (Ochoa y Aragón, 2007; Pérez, 2010; Gómez-Contreras, 2011; Gallego, García y Rodríguez, 2013) y de postgrado (Pereira y di Stefano, 2007; Sabaj, 2009, Castelló, González e Iñesta, 2010).

Sorprende, no obstante, que el incremento de la investigación en este campo haya descuidado sobremanera indagar en el manejo de la escritura por personas con síndrome de Down. En efecto, se han dado a conocer diferentes estudios que revelan diversos aspectos relacionados con el lenguaje oral (Díaz-Itza y Miranda, 2007; Galeote, Soto, Sebastián, Rey y Checa, 2012; Jiménez, Vicente, Sánchez, López y Rodríguez, 2013) o el proceso lector de este colectivo (Robles, 2010; Troncoso y Flórez, 2011; Bosch y Fernández-Llebrez, 2014); sin embargo, las investigaciones sobre el lenguaje escrito son muy escasas y se desconocen estudios realizados, específicamente, con personas con síndrome de Down en los que se 
analicen los procesos psicológicos implicados en la expresión escrita, lo que justifica la necesidad de esta investigación en el marco de los trabajos actuales.

En este sentido, el trabajo que se presenta analiza uno de los procesos cognitivos más relevantes de la composición escrita (la planificación) para conocer qué percepciones tienen las personas con síndrome de Down acerca de su competencia para planificar un texto, y a sabiendas de que el correcto aprendizaje de la escritura exige también decidir sobre el tipo de texto que se va a escribir para incorporar las características de ese género textual. Esta elección se justifica por ser la planificación uno de los elementos centrales del proceso escritor (Hayes y Nash, 1996; McCutchen, 2006).

Teniendo en cuenta lo anterior, los objetivos de esta investigación fueron: 1) desvelar qué operaciones de planificación manejan las personas con síndrome de Down cuando construyen un texto; 2) identificar posibles disfunciones en la planificación textual; 3) proponer algunas orientaciones para la mejora de la planificación textual. Ahora bien, dado el carácter exploratorio del estudio, y de acuerdo con los objetivos planteados, las hipótesis iniciales se formularon como interrogantes básicas acerca de las operaciones cognitivas implicadas en la planificación de textos: a) ¿Las personas con síndrome de Down conocen y utilizan el proceso de planificación textual?; b) ¿Qué operaciones cognitivas activan durante la planificación de un texto?; c) ¿Qué dificultades reconocen?

\section{Referente teórico}

El dominio de la lengua escrita (lectura-escritura) se percibe como un aprendizaje esencial para la adquisición de muchas de las competencias curriculares en los distintos niveles educativos (Rodríguez-Parra, Buiza, Adrián y Alegría, 2012). Sin embargo, la escritura es algo más que la producción mecánica de frases correctas, un proceso que reclama pensamiento, creación y resolución de problemas (Lensmire, 1994). Cuando se escribe, se requieren estrategias para resolver el problema, por lo que el escritor debe identificarlo, primero, y adoptar las medidas apropiadas, después (McCutchen, 2006). Esto exige comparar la representación del texto real con la representación del texto previsto, si bien esto último es muy complejo, especialmente para los aprendices más noveles (Bereiter y Scardamalia, 1987).

En la actualidad, la expresión escrita se percibe como una habilidad muy compleja en la que intervienen múltiples y diferentes procesos y subprocesos cognitivos y de otro orden (Kellog, 1994), los cuales no siguen una secuencia lineal de etapas, sino que poseen un carácter recursivo e interactivo, y se activan de acuerdo con las necesidades y peculiaridades 
del proceso escritor (Alamargot y Chanquoy, 2001). A este efecto, la enseñanza de la escritura ha sido abordada desde diferentes perspectivas y enfoques que responden a diferentes modelos teóricos en los que se privilegian ciertos aspectos y se enfatizan heterogéneos tipos de actividades. Sin embargo, entre los modelos de producción textual, por su innegable proyección didáctica, destaca el modelo de Hayes y Flower (1980), posteriormente actualizado (Hayes, 1996), este esconsiderado actualmente como el modelo que mejor explica la escritura de textosy el cualconstituye el marco de esta investigación. Hayes y Flower (1980) propugnan un modelo cuyos postulados suponen una base importante para los modelos teóricos posteriores (Alamargot y Chanquoy, 2001), además de ser un referente obligado en los estudios sobre la composición escrita, en tanto que proceso de resolución de problemas en que el escritor activa estrategias de planificación, análisis e inferencia, las cuales están condicionadas por variables externas e internas al propio sujeto.

De acuerdo con Hayes y Flower (1980), la escritura se describe como un proceso independiente de toma de decisiones que está orientado por las metas del escritor cuando redacta un texto, en el que múltiples operaciones mentales (planificación-transcripciónrevisión) se aplican a la tarea según los propósitos de la escritura. Hayes (1996) considera la escritura como un acto comunicativo que se desarrolla en un contexto socio-cultural, una actividad intelectual que requiere motivación y demanda procesos cognitivos y memoria. En este sentido, el modelo de Hayes muestra una descripción de la estructura de la producción escrita, incorporando elementos relevantes como la memoria de trabajo, la motivación y los procesos cognitivos de reflexión e interpretación. Se subraya el carácter no lineal de la escritura y se redimensiona la interrelación entre el contexto social (audiencia, colaboradores) y el contexto físico (texto producido, medio de elaboración) de la composición escrita.

En dicho modelo se diferencian dos componentes esenciales: el sujeto y el contexto de la tarea. En el primer componente se incluye la motivación, la afectividad, los procesos cognitivos, la memoria a largo plazo (MLP) y la memoria de trabajo. Se tienen en cuenta, pues, aspectos vinculados con las creencias y actitudes del escritor, predisposición hacia la tarea, establecimiento de metas, etc. $Y$ también los tres procesos psicológicos básicos, aunque renombrados: reflexión (planificación), producción (transcripción) e interpretación (revisión). E segundo componente comprende dos dimensiones básicas: contexto social (audiencia y personas del contexto) y contexto físico (textos escritos o leídos por el sujeto e instrumento con el que se escribe). Ambos contextos delimitan la actividad escritora. 
No obstante, el foco de atención de este modelo es el sujeto, su memoria de trabajo, su capacidad para hacer eficientes los procesos de codificación y descodificación de la informaciónasí como otros aspectos o componentes referidos a la persona que escribe: la afectividad, la motivación, la memoria a largo plazo (MLP) y los procesos cognitivos (reflexiónplanificación, producción-transcripción, e interpretación-revisión), en torno a los cuales se articula el proceso de la composición escrita.

La planificación de la escritura o proceso de reflexión (Hayes, 1996) -objeto de esta investigación- se concibe como la representación mental de la composición, en la que se incluyen todos los elementos del texto: contenido y forma estructural, sentido textual e intención escritora. Durante la planificación, el escritor contempla los objetivos que pretende, genera ideas y organiza los datos que son relevantes para la tarea. Por tanto, este proceso comprende diversos subprocesos: 1) la génesis de contenidos (ideas), que supone la búsqueda de informaciones, ya sean perfectamente estructuradas o no, en la MLP y/o fuentes externas de documentación; 2) la organización y estructuración de contenidos, o sea, una vez que las ideas se han obtenido, corresponde a otro subproceso la estructuración y organización de las mismas, con el fin de otorgar mayor coherencia al texto; 3) la formulación de objetivos, acción necesaria para controlar el acto de la composición escrita.

En la "génesis de contenidos" (ideas) hay que considerar dos procesos: a) la búsqueda en la memoria, para determinar la viabilidad de la información; b) la búsqueda heurística o referida a metas, para dirigir los hallazgos en la memoria hacia áreas fecundas de contenido (Scardamalia y Bereiter, 1986). La "estructuración de contenido" tiene como finalidad crear la estructura de una composición (organizar los contenidos). Las estructuras formales del texto servirán para guiar al escritor en la construcción del texto y al lector en la comprensión deeste. Por último, la "determinación de objetivos" sirve para controlar el acto de la composición. Los escritores expertos poseen un sistema bien definido de objetivos principales y secundarios, y tienen desarrolladas las estrategias que les permiten recuperar dicho sistema en la memoria a corto plazo. Se admite que un escritor estratégico es aquel que puede dirigir competentemente las dificultades de escritura, posee creencias positivas de auto-eficacia y está satisfecho con su rendimiento en tareas escritas (Hidi y Boscolo, 2006). El escritor novel, sin embargo, no construye un plan bien definido y los objetivos le suponen una carga adicional en la memoria a corto plazo. Además, es probable que no utilice las estrategias idóneas o no disponga de ellas para recuperar los objetivos o para traducirlos en ideas; por tanto, mantendrá creencias bajas de auto-eficacia y sentirá ansiedad o aprensión hacia la escritura (Hidi y 
Boscolo, 2006). Lo más común, no obstante, es que quien escribe elabore uno o varios "pretextos" antes del que emita como definitivo. El texto representa una actualización de un acto discursivo, determinado por la intención comunicativa.

La transcripción o producción textual es la función de elaborar un producto escrito a partir de representaciones internas construidas en el contexto de la tarea. Consiste en dar forma lingüística a las ideas (contenido), para lo cual el escritor activa sus conocimientos previos y pone en práctica ciertas habilidades: a) gramaticales (ortografía, léxico, sintaxis...); b) convenciones sociales (tipografía, formato...); c) cohesión discursiva. El proceso de traducción tiene lugar cuando se obtiene el material de la memoria bajo la orientación de un plan y se convierte en texto. En este proceso, los escritores noveles atienden más a los aspectos de bajo nivel cognitivo, de tal manera que sus transcripciones suelen ser una mera copia de lo que tienen en la mente y de lo que expondrían de forma oral (Scardamalia y Bereiter, 1992). No obstante, la transcripción es un proceso complejo, dado que implica transformar unas ideas, organizadas en el espacio abstracto de la memoria a corto plazo e interconectadas en una redacción escrita lineal con unos símbolos que reflejen, lo más fielmente posible, lo que se tenía en la mente.

Por último, la revisión o interpretación (Hayes, 1996) conlleva un conjunto de operaciones de carácter cíclico o recursivo: a) repasar; b) evaluar, c) identificar problemas, d) corregir, d) re-evaluar. La revisión implica lectura, evaluación textual y reescribir (McCutchen, 2006). Es un proceso flexible que puede repetirse todas las veces que el escritor estime conveniente. Asimismo, es un proceso complejo, por cuanto implica una evaluación del texto para modificar aquellos aspectos en los que el escritor percibe algún desajuste en relación con lo que tenía planificado o en relación con las reglas gramaticales y ortográficas. De ahí que la interpretación (revisión) es una función que crea representaciones internas, a partir de estímulos lingüísticos y gráficos, y que requiere de una experiencia considerable. La revisión, lejos de ser una actividad rápida, es una acción en la que el escritor necesita tiempo para examinar el producto escrito e introducir las mejoras que estime oportunas. En la revisión, la lectura juega un papel fundamental para detectar los problemas y/o errores (Hayes, 1996) ya que el escritor vuelve de un modo global y específico sobre su texto para apreciar el grado de acomodo de este a la pretendida situación comunicativa en la que está participando, para revisar y corregir, en su caso, cuestiones puntuales o de mayor trascendencia, por lo que no hay que excluir que se genere un nuevo proceso de escritura a partir de este proceso. 
En la investigación sobre la composición escrita se han manejado tres enfoques que pueden y deben ser complementarios (Berninger y Whitaker, 1993), y cuya diferencia reside, principalmente, en su objeto de estudio: producto, proceso y ecológico (Castelló y Monereo, 1996; Torrance, Thomas y Robinson, 2000; Salvador, 2008). El enfoque de producto, de orientación conductista constituye la perspectiva más tradicional en el estudio de la escritura y su apogeo se sitúa en la década de los setenta. En este caso, el centro de atención es una conducta observable y medible que, en el caso de la escritura, es el texto escrito: sus aspectos formales (grafía y ortografía) y estructurales (significado del texto y cohesión textual). Desde este enfoque, se enfatiza una perspectiva estrictamente lingüística en la que sobresalen las características formales del texto. Su aceptación en el contexto educativo derivó de su efectividad para evaluar la organización sintáctica, el léxico, la morfología, la ortografía y la puntuación en la escritura.

En el enfoque de proceso -seguido en esta investigación- se supera la evaluación sumativa de la escritura, basada principalmente en la corrección de errores gramaticales superficiales del texto como producto (Applebee, 1981), para analizar el proceso de composición escrita (Levy y Olive, 2002; Bayat, 2014). Este enfoque, que se consolida en los años ochenta, forma parte de los planteamientos que subyacen en la investigación mediacional-cognitiva o interpretativa. El conocimiento de los procesos cognitivos que intervienen en la escritura puede intuirse como una tarea compleja, derivada tanto de la dificultad de los procesos mentales, en general, como del proceso de escritura, en particular, siendo la relevancia de sus resultados muy atractiva para la investigación.

En este enfoque ocupan un lugar destacado aspectos tales como (Marinkovich, 2002): a) el descubrimiento del propio pensamiento del autor; b) el proceso de escritura regido por metas, en el que la planificación es una fase esencial; c) la retroalimentación durante el proceso escritor, a través de la pre-escritura y la elaboración de múltiples borradores. Se cambia, por tanto, la perspectiva de la enseñanza y se subraya la importancia de escribir sobre temas de interés para el estudiante, la necesidad de planificar la redacción como una actividad contextualizada con un propósito específico, la utilidad de las tareas de pre-escritura y de los borradores, la importancia de escribir para una audiencia que reacciona ante el texto y la importancia del contenido y el proceso de expresión con respecto a la gramaticalidad del producto final. A partir de entonces, los aprendices deben ser conscientes de los diferentes componentes del proceso de redacción que, lejos de percibirse como lineal, habría de contemplarse como recursivo. 
El enfoque contextual surge a partir de la década de 1980 en EE.UU. Se le conoce también como enfoque ecológico porque está basado en la perspectiva etnográfica de la escritura al considerar la composición escrita como un proceso estrechamente vinculado y determinado por el contexto en el que se desarrolla (Camps, 1997). La escritura de un texto es, pues, un proceso situado y subsidiario de una determinada situación de comunicación (Camps y Castelló, 1996; Pereira y di Stefano, 2007), que exige entender cuáles son los procesos que activan los aprendices durante el acto de la composición para propiciar actividades que incentiven el desarrollo mismo de la alfabetización (Vygotsky, 1986). A través de la interacción social, los aprendices adquieren información y habilidades relacionadas con la escritura y desarrollan nuevas maneras de representar el conocimiento interno (Sperling y Freedman, 2001). El objetivo de este enfoque es, para García y Salvador (2010), analizar las situaciones educativas concretas a partir de la necesidad de profundizar en la dimensión social y comunicativa de la escritura para plantear modelos alternativos de enseñanza acordes con esta dimensión.

Pese a su complejidad intrínseca y, como ya se ha señalado, en esta investigación se adopta primordialmente el enfoque de proceso por su indudable relevancia para comprender en profundidad los procesos de la escritura, dado su carácter explicativo e interpretativo.

\section{Metodología}

Se trata de una investigación exploratoria y fundamentalmente cualitativa, de estudio de caso colectivo (Stake, 2010) en la que se utilizó la entrevista cognitiva para obtener los datos y el análisis de contenido para interpretarlos. Los estudios exploratorios sirven para familiarizarnos con fenómenos relativamente desconocidos y obtener información sobre la posibilidad de llevar a cabo una investigación más completa (Hernández, Fernández y Baptista, 2003). A su vez, el estudio de casos, como diseño cualitativo que analiza "la particularidad y la complejidad de un caso singular, para llegar a comprender su actividad en circunstancias importantes" (Stake, 2010, p.11), ha de permitirnos alcanzar la comprensión de la realidad objeto de estudio. La intención es obtener, mediante el análisis en profundidad del caso, suficientes evidencias de las que inferir posibles conclusiones en función de los objetivos planteados (Gillham, 2000). 


\subsection{Participantes}

Participaron voluntariamente en el estudio -que aborda de forma comprensiva el proceso de planificación de la escritura en cuanto competencia muy relevante para el desarrollo integral y la autonomía de las personas que presentan síndrome de Down-, previa petición, 25 estudiantes (10 alumnas y 15 alumnos) escolarizados en tres centros específicos de Educación Especial ubicados en dos ciudades de la Comunidad Autónoma de Andalucía (España). Su edad oscila entre los 16 y los 21 años (media de 19.4). Todos ellos son capaces de mantener una sencilla conversación y de leer y escribir textos muy sencillos ya que poseen un nivel de lectoescritura cercano a un estudiante de segundo ciclo de Educación Primaria. Los criterios de inclusión fueron: a) documentación citogenética de la trisomía-21; b) ausencia de déficits neurosensoriales evidentes, tales como los auditivos y visuales, y c) ser capaces de leer y escribir textos sencillos.

Conviene insistir, no obstante, que este grupo se considera como un caso, sin detrimento de que puedan detectarse diferencias intra-caso. Las diferencias individuales pueden reflejar no solo heterogéneos estilos de escritura, sino diferentes conocimientos, habilidades y competencias sobre el proceso escritor. El caso, sin embargo, se considera una totalidad única e integrada, con sus propios límites físico, social o temporal que le confieren entidad, es por tanto un sistema acotado (Ceballos, 2009).

\subsection{Instrumentos}

Para obtener los datos se ha optado por el instrumento más utilizado en la investigación cualitativa, y mucho más en los estudios de caso (Yin, 2003): la entrevista semiestructurada a partir de un cuestionario-guía (Cfr. Salvador, 2005, pp. 62-66) que ofrece pistas a las y los estudiantes sobre las operaciones o procesos que supuestamente se activan durante la composición de un texto, y les ayuda a reflexionar y verbalizar lo que hacen para colegir los procesos ejecutivos de la escritura y sus posibles relaciones. El objetivo de la entrevista semiestructurada, en estos casos, es la exploración de los conocimientos del alumnado sobre determinados fenómenos escriturales y de los procesos que realiza para construirlos (Guasch y Ribas, 2013).

Esta técnica no difiere significativamente de otra técnica informal ("pensamiento en voz alta") que consiste en reclamar la verbalización de lo que ha pensado y hecho un sujeto inmediatamente después de escribir un texto. Sin embargo, dadas las peculiaridades del alumnado, se ha preferido en este estudio proporcionar algunas pistas, en forma de preguntas, 
acerca de las operaciones y procesos que los estudiantes han podido seguir durante la elaboración de un texto para facilitarles la tarea de verbalización. Se pretende enriquecer las posibilidades de análisis de la escritura para detectar los procesos que ocultan los métodos de investigación que solo examinan el producto escrito.

La utilización del pensamiento en voz alta para obtener datos ha demostrado ser una herramienta útil a la hora de captar lo que sucede en la mente del escritor individual (Hayes y Flower, 1980) y permite a investigadoras e investigadores analizar cuáles son las funciones cognitivas y metacognitivas que operan en la mente de quien escribe.

El cuestionario utilizado para realizar la entrevista (Tabla 1) fue validado por el procedimiento de "juicio de expertos" y triangulación (Fox, 1981; Hodder, 2000). Tres investigadoras y dos investigadores, expertos en Didáctica de la Lengua determinaron la validez de su contenido (consenso unánime), de acuerdo con criterios de representatividad y relevancia de los ítems.

Tabla 1

Síntesis del cuestionario-guía sobre planificación

\section{A. Génesis de ideas}

1) Antes de escribir un texto ¿piensas lo que vas a escribir?

2) Cuando vas a escribir ¿piensas en palabras relacionadas con el tema?

3) ¿De dónde sacas las ideas: de la cabeza...?

4) Cuando vas a escribir ¿buscas palabras o simplemente las que recuerdas?

5) ¿Las anotas?

6) Las palabras o ideas que recuerdas ¿las escribes todas o eliges algunas?

7) ¿Anotas en una hoja aparte lo que quieres poner en el texto, antes de escribirlo?

8) ¿Usas alguna fórmula para recoger y ordenar las ideas y no olvidarlas?

9) En el momento de escribir ¿escribes lo que se te ocurre o tienes en cuenta lo que pensaste antes de escribir?

10) Cuando escribes un texto ¿encuentras las palabras adecuadas?

11) Las palabras que finalmente escribes ¿son las que realmente querías?

12) ¿Sabes cómo y dónde encontrar nuevas palabras e ideas para escribir?

\section{B. Formulación de objetivos}

13) ¿Por qué sueles escribir un texto?

14) Antes de escribir ¿piensas en la persona que lo leerá?

15) Cuando escribes ¿lo haces para que te entiendan?

16) ¿Qué quieres conseguir cuando escribes un texto?

17) ¿Qué haces para conseguirlo?

18) ¿En qué parte del texto se puede notar?

\section{Organización o estructuración del contenido}

19) Antes de escribir ¿clasificas u ordenas las ideas?

20) ¿Cómo consigues escribir un texto? ¿Cómo las ordenas? ¿qué haces?

21) ¿Por qué las ordenas así y no de otra forma?

22) ¿Utilizas alguna fórmula para organizar las ideas?

23) ¿Piensas las frases completas o las escribes como se te van ocurriendo?

24) Antes de escribir ¿piensas en el tipo de texto? Por ejemplo: expositivo, narrativo, descriptivo...

25) ¿Ordenas las ideas y las palabras de forma distinta, según el texto?

26) ¿Sabes las partes que debe tener tu texto?

27) ¿Sigues alguna regla?

Fuente: Elaboración propia del autor (2015) 


\subsection{Procedimiento}

Para facilitar la verbalización de los estudiantes sobre los procesos implicados en la planificación de las tareas de escritura, se les solicitó la escritura de un cuento o historia sobre algún hecho o suceso de su interés para que, inmediatamente después, evocaran las operaciones manejadas durante su elaboración. La elección del texto narrativo se justifica por (a) ser uno de los modos del discurso monogal que funciona como esquema de comprensión y de interpretación más básico (Calsamiglia, 2000); (b) ser utilizado por el niño desde una edad muy temprana como forma de comprender el mundo (Bruner, 2004); (c) ser el que más frecuentemente elaboran los estudiantes libremente (Salvador, 2008).

Todas las entrevistas fueron grabadas y transcritas literalmente con posterioridad con la ayuda de un transcriber (aparato técnico de reproducción controlada de los registros de las entrevistas) y un procesador de textos. Así, el discurso oral se transformó en texto escrito sobre el que se realizó el análisis de contenido por medio del cual se clasificó la información siguiendo un sistema de categorías (Bardín, 1986; Krippendorff, 2002). En efecto, se dividieron los textos en unidades de contenido teniendo en cuenta unas categorías que nos permiten reunir mucho material en unidades de análisis más significativas (Miles y Huberman, 1994). Igualmente, las dimensiones psicolingüísticas implicadas en la composición escrita se agruparon para facilitar el análisis y la interpretación de los datos en aquellas categorías que se corresponden con las operaciones que los estudiantes realizan durante la planificación de un texto, momento en el que el y la estudiante muestran realmente sus habilidades escritoras, por cuanto esta operación incluye potencialmente todo el proceso escritor (Graham y Harris, 2005).

Desde esta perspectiva, la planificación o reflexión (Hayes, 1996) es considerada como el borrador mental de la composición, un proceso en el que la persona piensa lo que va a escribir, establece y prioriza los objetivos y organiza las ideas, lo cual permite establecer unas categorías u operaciones básicas en cuya definición se incorporan todos sus matices. A las categorías se asignaron códigos representados por letras mayúsculas y números (Tabla 2). 
Tabla 2

Sistema de categorías para analizar los protocolos de las entrevistas

\begin{tabular}{|c|c|c|}
\hline CATEGORÍAS & cóDIGOS & DEFINICIÓN \\
\hline Génesis de ideas & $(+-\mathrm{P} 1)$ & $\begin{array}{l}\text { Capacidad de quien escribe para producir las ideas necesarias que le } \\
\text { permitan la construcción de un texto. }\end{array}$ \\
\hline Auditorio & (+-P2) & $\begin{array}{l}\text { Capacidad de quien escribe para pensar en los destinatarios personas } \\
\text { que leerán el texto. }\end{array}$ \\
\hline Objetivos & (+-P3) & $\begin{array}{l}\text { Capacidad de quien escribe para formular las finalidades, intenciones } \\
\text { y objetivos que guían la elaboración de un texto. }\end{array}$ \\
\hline Selección de ideas & $(+-\mathrm{P} 4)$ & $\begin{array}{l}\text { Capacidad de quien escribe para identificar selectivamente las ideas } \\
\text { principales de un futuro texto. }\end{array}$ \\
\hline Ordenación de ideas & $(+-P 5)$ & $\begin{array}{l}\text { Capacidad de quien escribe para organizar/secuenciar } \\
\text { adecuadamente las ideas de un texto. }\end{array}$ \\
\hline Fuente de ideas & $(+-\mathrm{P} 6)$ & $\begin{array}{l}\text { Capacidad de quien escribe para identificar y seleccionar los recursos } \\
\text { de los cuales extraer los contenidos de un texto. }\end{array}$ \\
\hline Registro de ideas & $(+-\mathrm{P} 7)$ & $\begin{array}{l}\text { Capacidad de quien escribe para utilizar instrumentos donde anotar } \\
\text { las Ideas (contenidos) de un texto, que le servirán como guía durante } \\
\text { su transcripción. }\end{array}$ \\
\hline Organización textual & (+-P8) & $\begin{array}{l}\text { Capacidad de quien escribe para utilizar la estructura textual } \\
\text { adecuada, según el tipo de texto. }\end{array}$ \\
\hline
\end{tabular}

Fuente: Elaboración propia del autor (2015).

Se ha de advertir, no obstante, que las categorías utilizadas derivan de planteamientos teóricos sólidos que gozan de suficiente validez y fiabilidad, como sucede con los acreditados modelos cognitivos sobre la expresión escrita de Hayes y Flower (1980) y Hayes (1996) ampliamente reconocidos por la comunidad científica. Además, este sistema categorial también fue validado por el procedimiento de "juicio de expertos" y "triangulación de codificadores independientes" (Fox, 1981; Stake, 2010), de tal manera que, según los expertos, reúne los requisitos necesarios para satisfacer las exigencias de esta investigación (Bardin, 1986): exhaustividad, exclusión mutua, homogeneidad, objetividad, pertinencia, productividad.

Finalmente, se agruparon los contenidos de similar código y se calcularon las frecuencias, los porcentajes y las medias para abordar la interpretación global de los datos, tanto numéricos como verbales, teniendo en cuenta que los datos cuantitativos se utilizaron para corroborar y apoyar los testimonios de los y las estudiantes.

\section{Resultados}

Como señalan las profesoras Ruiz y Camps (2009), los estudios de corte cualitativo pueden ser auxiliados por análisis cuantitativos en ciertos aspectos y los datos cuantitativos, interpretarse con ayuda de observaciones cualitativas. Por ello, los resultados del proceso de 
planificación se obtuvieron del análisis de contenido de las entrevistas al alumnado con síndrome de Down así como de la cuantificación y valoración de sus respuestas. En este sentido, las frecuencias positivas se corresponden con la adecuada ejecución de la operación a la que se refiere cada categoría (sumando el número de veces que los estudiantes declaran utilizar adecuadamente esa estrategia), mientras que las frecuencias negativas indican la no realización o inadecuación de la operación indicada (sumando el número de veces que la estrategia es ignorada, no utilizada o manejada inadecuadamente).

En el análisis cuantitativo (Tabla 3) se apreciaron valores más elevados de frecuencias positivas en la mayoría de las categorías, de lo cual puede inferirse inicialmente lo siguiente:

1. El alumnado con síndrome de Down entrevistado realiza las operaciones del proceso de planificación de la escritura en el $54.91 \%$ de los casos.

2. Estos estudiantes no realizan o, lo hacen de forma inadecuada, en un $45.09 \%$ de los casos, algunas de las operaciones de planificación.

3. El registro de ideas, la ordenación de estas y la organización textual son las operaciones cognitivas en las que se apreciaron los mayores inconvenientes.

4. El registro de ideas se reveló como la operación más complicada para estas personas.

Tabla 3

Frecuencias de las categorías de planificación

\begin{tabular}{llcccc}
\hline \multicolumn{1}{c}{ Categorías } & Códigos & Frecuencias + & Frecuencias - & Total (+-) & $\%$ \\
\hline Génesis de ideas & $(+\mathrm{P} 1 /-\mathrm{P} 1)$ & 68 & 13 & 81 & 12.0 \\
Auditorio & $(+\mathrm{P} 2 /-\mathrm{P} 2)$ & 34 & 16 & 50 & 7.4 \\
Objetivos & $(+\mathrm{P} 3 /-\mathrm{P} 3)$ & 74 & 20 & 94 & 13.9 \\
Selección de ideas & $(+\mathrm{P} 4-\mathrm{P} 4)$ & 44 & 4 & 48 & 7.1 \\
Ordenamiento de ideas & $(+\mathrm{P} 5 /-\mathrm{P} 5)$ & 33 & 114 & 16.9 \\
Fuente de ideas & $(+\mathrm{P} 6 /-\mathrm{P} 6)$ & 65 & 82 & 71 & 10.5 \\
Registro de ideas & $(+\mathrm{P} 7 /-\mathrm{P} 7)$ & 14 & 81 & 118 & 17.5 \\
Organización textual & $(+\mathrm{P} 8 /-\mathrm{P} 8)$ & 37 & & & \\
\hline Total de Frecuencias & & 369 & 303 & 672 & 100 \\
\hline
\end{tabular}

Fuente: Elaboración propia del autor (2015). 
Al tratarse de un estudio de caso colectivo, para la correcta interpretación de los datos, conviene insistir en que el número de frecuencias (Gráfico 1) se refiere al conjunto de los veinticinco participantes en la investigación. No obstante, para alcanzar los significados de este estudio, problema fundamental en el estudio de casos, se han utilizado dos estrategias: la interpretación individual de los datos y su suma integrada para poder explicar algo sobre el conjunto (Stake, 2010). Pero el interés de este estudio no está tanto en la cuantificación de los datos cuanto en el análisis en profundidad de las entrevistas. Para ilustrar esta interpretación, se incluyen algunas manifestaciones literales de los estudiantes y se muestran los resultados e interpretaciones de cada una de las operaciones específicas de la planificación.

\section{Gráfico 1}

Frecuencias positivas versus negativas de la planificación

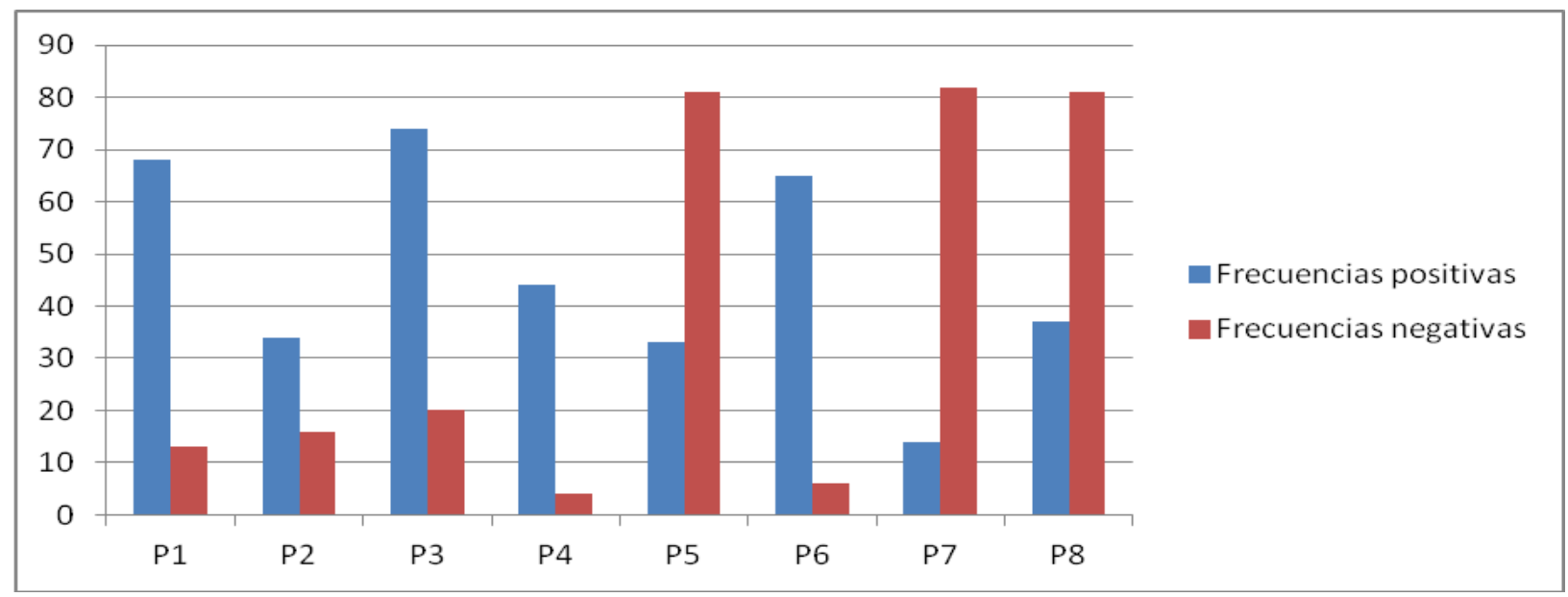

Fuente: Elaboración propia del autor (2015).

Sobre la génesis de ideas (+-P1)

El análisis cualitativo de las entrevistas pone de manifiesto que las personas con Síndrome de Down dedican algún tiempo a pensar en los contenidos de sus textos antes de iniciar una redacción.

$P:$ Antes de escribir un texto o una redacción ¿piensas lo que vas a poner?

R: Sí lo he pensado..., aunque me ha costado algo... (S3, S4, S6, S8). Siempre lo pienso y después escribo lo que pienso... (S1, S2, S7, S10, S11, S13). Sí, sí que lo pienso... (S12, S14, S17, S24). Lo primero que hay que hacer es pensar para después poderlo hacer lo que puedas... (S25). ... cuando estoy escribiendo, poco a poco pienso... (S16, S22). 
Sin embargo, algunos admiten que no siempre piensan antes de escribir: ... no lo sé, pongo lo que se me ocurre en el momento... (S5). No, lo voy poniendo según me va saliendo (S9). Yo según me vienen a la cabeza (S20). No sé muy bien, la verdad... (S23).

Otros sostienen que, cuando escriben un texto, procuran emplear siempre las palabras que poseen una mayor relación con el tema de escritura.

$\mathrm{P}$ : Antes de escribir un texto o una redacción ¿piensas las palabras que están relacionadas con el tema?

R: Sí, para no cometer muchos fallos en el texto... (S4, S10, S16). Sí, sí pienso en palabras que tengan que ver para escribir bien (S1, S3, S18).

Tampoco faltan respuestas reveladoras de las contradicciones en las que caen estos estudiantes cuando tienen que realizar tareas de escritura.

P: En el momento de escribir ¿escribes lo que se te va ocurriendo o tienes en cuenta lo que pensaste antes de escribir y anotaste en una hoja aparte?

$\mathrm{R}$ : Yo escribo lo que me viene a la mente, a la cabeza (S16, S22, S23). Yo voy escribiendo y pensando poco a poco (S21).

Sobre el auditorio (+-P2)

Las respuestas de los estudiantes reflejan mayoritariamente que, cuando elaboran un texto, suelen tener en cuenta a los destinatarios de sus escritos y tratan de acomodarse a ellos. Algunos estudiantes admiten, no obstante, que no piensan en los receptores de sus textos a quienes ignoran o incluso desconocen.

$\mathrm{P}:$ Antes de escribir un texto o una redacción ¿piensas en la persona que lo va a leer?

R: Sí, sí, siempre... (S16, S18, S19, S23, S24, S25). Sí, siempre lo hago para que lo entienda... (S1, S2, S4, S14, S15). Sí, y espero que esté bien (S6, S8, S12, S13). Pues claro, lógicamente (S3, S10, S11, S17).

R: No, porque no lo sabía (S3). No he pensado en eso (S9). Que lo entiendan mis amigos (S5, $\mathrm{S} 7, \mathrm{~S} 16)$. No sé... ¿que una persona lo entienda? (S22). 
Los hay quienes responden de forma ambigua: unas veces sí, otras no (S20). Sí, porque yo lo entiendo (S21).

Sobre los objetivos (+-P3)

El análisis cualitativo de las entrevistas revela que a los estudiantes los guía una heterogeneidad de objetivos cuando se enfrentan a tareas de expresión escrita, aunque admitan no saber muy bien dónde podría apreciarse esa intencionalidad en sus escritos. En efecto, por motivaciones diversas, unos declaran que escriben para comunicarse 0 entretenerse, otros señalan que lo hacen por placer o divertimento y otros para conocer sus habilidades en el dominio de la gramática.

$\mathrm{P}$ : Cuando escribes un texto o una redacción ¿qué quieres conseguir?

R: Que me quede bien y me guste... (S1, S6, S16). Yo... comunicarme con los demás (S8, S9, S17). Porque me gusta escribir y que alguien se entere... (S10, S13, S18). Para contar mis vacaciones... (S5, S7, S21). Para que se rían, porque me equivoco mucho y... (S16). Hacerlo bien... tenerlo bien... hacer buena letra y que esté bien... poner bien todo, las palabras bien escritas (S2, S3, S12, S21, S22). No cometer faltas... (S14, S15, S21).

A veces, el alumnado declara no saber cuál es la finalidad de su escrito, por lo que no siempre sus textos están guiados por objetivos concretos: no sé (S3, S11). Eso no lo sé (S25). No entiendo, ni idea (S21). No quiero conseguir nada... terminar (S4).

Sobre la selección de ideas (+-P4)

Los estudiantes declaran que, en la elaboración de sus textos, incorporan todo lo que se les ocurre en el momento de la redacción, así como aquellas ideas que previamente han pensado en relación con el tema de escritura.

P: Cuando vas a escribir un texto o redacción ¿buscas ideas o simplemente las que se te vienen a la mente?

R: Yo pongo lo que se me va ocurriendo... pongo lo que me viene (S1, S2, S5, S16, S17). Todo lo que he pensado... (S7, S9, S13). Escribo y según me sale... lo que pienso entero... (S11). Suelo seleccionar lo que más me gusta... lo que mejor suena (S14, S21, S23). Yo pienso y elijo algunas (S18, S20, S24). 
A veces, las respuestas de los estudiantes revelan la ejecución simultánea de dos operaciones cognitivas («selección de ideas» y «registro de ideas»): las dos cosas... pero si se me ocurre algo pues lo pongo... (S15). Lo que se me va ocurriendo... y también pienso (S12). También cojo lo que había escrito antes en una hoja (S19).

Sin embargo, en ocasiones, algunos estudiantes ofrecen respuestas contradictorias o, cuando menos, dubitativas, que impiden conocer el manejo real de esta operación. Por ello, como se señala en la prospectiva de la investigación, contrastar las declaraciones de estos estudiantes con el análisis de sus productos (textos) hubiese sido muy ilustrativo.

P: Cuando estás escribiendo un texto ¿escribes todas las palabras que se te ocurren, algunas o las que ya habías pensado?

R: Escribo según me sale. Lo que ya he pensando... (S3, S16). Escribo todo (S4, S10, S22). Sí, bueno, no lo sé (S20).

Sobre la ordenación de ideas (+-P5)

El análisis de las entrevistas evidenció que estos estudiantes eluden organizar previamente las ideas que van a plasmar en sus textos, ya sea de forma intencional o por simple desconocimiento. Mayoritariamente, declararon ignorar este proceso de planificación textual. Sorprende, sin embargo, que algunos estudiantes manifestaran que sí organizan de forma global sus escritos, habida cuenta de las repercusiones positivas que tiene para la calidad de sus textos.

P: Antes de escribir un texto ¿clasificas u ordenas de alguna forma las palabras que vas a escribir?

R: No, no. Seguro que no (S2, S12). Es que no entiendo, no ordeno... (S3, S11, S16). Yo de eso no hago nada... (S7). No, ¿para qué?... ¿para qué sirve? (S5). ¿Es que hay que hacer eso? (S4, S20). Nunca me lo han dicho (S6, S10). Que yo escribo como se me ocurre... (S17, S21). Yo no las ordeno... (S9, S13, S16).

R: Sí lo pienso antes, las ordeno... (S1). Las clasifico y las ordeno... si no me lío mucho, no me queda bien... (S18). Siempre me gusta ponerlas con un cierto orden... así me ayudan... (S19). Sí, las clasifico (S24). 
Otra cosa es cuando se les pregunta qué es lo que hacen exactamente para ordenar las ideas de un escrito. Entonces contestan negativamente o declinan responder: yo no sé, qué sé yo de eso, pues dímelo tú (S1, S18, S19, S24).

También han dejado claro con sus respuestas que jamás acuden al uso de esquemas o trucos para que les ayuden en la elaboración de sus textos o escritos.

$P:$ ¿Utilizas algún esquema o cuadro para organizar las ideas?

R: No (S2, S6, S9, S10, S14, S22). No sé hacer eso (S4, S5, S11, S12). Que no ordeno nada (S8, S13, S15, S15, S21). ¿Cómo se hace? (S7, S20).

Sobre la fuente de ideas (+-P6)

En general, los análisis desvelaron que estos estudiantes suelen obtener las ideas para elaborar sus textos en diferentes fuentes, siendo su propia imaginación, internet y algunos libros los principales recursos manejados para obtener ideas.

P: ¿De dónde sacas las ideas para escribir un texto o una redacción?, ¿conoces sitios de donde sacar ideas para poner en tus textos?

R: De lo que leo que me acuerdo... (S6, S8, S9, S19, S24). De los libros que tengo... miro en los libros... (S16, S18, S20, S21). A mí me gusta mucho buscar en internet (S7, S13, S17). De la cabeza, se me vienen a la mente, me acuerdo... (S5, S18). Busco en mi cabeza... me vienen a la cabeza (S20, S25). De lo que he pensado yo (S5, S10, S12, S14).

Algunos estudiantes admiten ignorar la existencia de fuentes o recursos para obtener ideas con las que enriquecer sus textos: No lo sé (S2, S23, S25).

Sobre el registro de ideas (+-P7)

A tenor de las declaraciones de los estudiantes, se puede afirmar que este colectivo confía más en su propia imaginación y evita, en general, el manejo de otros recursos en el momento de elaborar sus escritos. En este sentido, no suelen recurrir a anotaciones previas ni a fórmulas o trucos que les ayuden a recordar ideas para incorporarlas a sus textos

P: ¿Usas alguna fórmula o truco para recoger y ordenar las ideas que se te ocurren y no olvidarlas mientras escribes? 
R: No uso truquillos... (S3, S4, S7, S13). No, nada, no uso nada (S5, S8, S9, S10, S16, S17). Yo lo ordeno en mi cabeza y ya está (S11, S15, S20, S22, S23). No anoto nada, lo escribo directamente en la libreta (S12, S14, S18, S21, S24).

Sin embargo, algunos estudiantes admitieron el manejo de esta estrategia cuando se enfrentan a la elaboración de un texto, como por ejemplo al utilizar borradores previos en los que anotan ideas que luego retoman para escribir: uso siempre un truco, escribo (S17, S19). Tomo notas (S17). Sí, en mi libreta (S25). Pero no fue menos cierto que quienes declararon utilizar alguna fórmula como ayuda entraron en contradicción cuando aseguran terminantemente que no tomaban notas previas que les ayudasen a recordar las ideas que supuestamente habían pensado: no, bueno no... pues la verdad es que no (S17, S19, S25).

\section{Sobre la organización textual (+-P8)}

En general, los estudiantes desconocen la estructura de los géneros discursivos según se desprende de los análisis cualitativos de las entrevistas. Además, ignoran la existencia de diferentes tipologías textuales y, por ende, su estructura, sin reparar en la importancia que tiene una adecuada ordenación de las ideas en función del tipo de texto.

P: Antes de escribir un texto o redacción ¿piensas qué tipo de textos vas a escribir?

R: Yo no (S2, S6, S10, S12, S14, S17). Siempre pongo lo que me gusta... no sé de qué texto hablas... (S1, S7, S9). ...no lo entiendo lo que me dices (S8, S13, S15, S20). Lo hago sobre la marcha... (S4, S5, S11, S16).

Llama la atención, sin embargo, que algunos participantes manifiesten respetar la secuencia temporal de principio-medio-final o que declaren poseer habilidades para diferenciar entre los hechos principales y los secundarios, desdeñando, inclusive, las ayudas para realizar un texto. Tal vez estos hallazgos se deban más a factores de didáctica (de enseñanza o pedagógicos) que a una condición o característica de aprendizaje de un colectivo en particular.

P: ¿Cómo consigues escribir un texto o una redacción?, ¿Cómo ordenas las ideas?, ¿Sigues el orden arriba-abajo, derecha-izquierda?, ¿Las ordenas en el tiempo?, ¿Distingues lo más importante de lo menos importante? 
R: Por supuesto, sí... (S19, S24). Distingo lo más importante de lo menos... (S21, S22, S25). Siempre ordeno las historias (S18). Lo cuento poco a poco y en orden (S21). Yo no necesito ayuda... me gusta y escribo (S18).

Ninguno de los estudiantes declaró manejar reglas mnemónicas para recordar las partes o estructura de un texto.

P: ¿Sigues alguna regla que te ayude a recordar las partes del texto?

R: No (S6, S10, S16, S17, S20, S22, S23). No sé (S2, S14, S20). ¿Para qué? (S18).

\section{Conclusiones y propuestas}

El objetivo de esta investigación fue desvelar cómo planifican la escritura un grupo de estudiantes con síndrome de Down, se identificaron las disfunciones que muestran y las habilidades que poseen. Desde esta perspectiva, este estudio de casos colectivo ha de dar respuesta a las tres cuestiones planteadas inicialmente y detalladas a continuación para establecer los principales aportes de esta investigación. Se debe señalar, no obstante, que los hallazgos obtenidos no son exclusivos del alumnado con síndrome de Down, sino que, como podrá observarse en las investigaciones citadas en la introducción de este artículo, algunos de estos hallazgos ya se han advertido en otros grupos de estudiantes.

¿Los participantes en este estudio conocen y utilizan el proceso de planificación textual?

En general, poseen un conocimiento muy superficial de cómo enfrentarse a la planificación de un texto. Aunque manifiestan utilizar algunas de las operaciones básicas del proceso de planificación se declaran inhábiles en el uso de otras muchas o se observan contradicciones que hacen dudar de que manejen de forma eficiente las estrategias requeridas.

¿Qué operaciones realizan durante la planificación de un texto?

El estudio puso de manifiesto que el alumnado participante, cuando se enfrenta a tareas de escritura, recurre a algunas estrategias cognitivas básicas:

- Suelen pensar antes de escribir para obtener ideas o palabras que tengan relación con el tema de escritura y les permita elaborar un texto de mayor calidad. 
- Aunque a veces ignoren a su audiencia, suelen pensar quiénes son los destinatarios de sus escritos para tratar de acomodarse a ellos.

- También, suelen recurrir a diferentes fuentes para obtener datos sobre el tema de escritura. Consideran que, además de la propia imaginación, se puede acceder a contenidos sobre un determinado tema consultando libros e incluso, internet

- Se mueven frecuentemente por motivaciones muy heterogéneas a la hora de enfrentarse a una tarea de escritura. El interés por comunicarse, por entretenerse o por mero placer son motivos que justifican su predisposición hacia la escritura. Incluso, algunos se han mostrado proclives hacia la escritura por el simple hecho de conocer sus propias habilidades como escritores.

- Sorprende que algunos de los estudiantes hayan manifestado como una de sus principales preocupaciones, a la hora de elaborar un texto, la buena caligrafía y la ausencia de faltas de ortografía.

¿Qué dificultades encuentran estos estudiantes?

Por último, el estudio reveló que este colectivo exhibe importes limitaciones durante el proceso de planificación de la escritura:

- No siempre son capaces de seleccionar las ideas o palabras que más relación presentencon el tema de escritura y, a veces, plasman en sus escritos lo que se les ocurre en el momento de la transcripción.

- Aunque sean conscientes de su trascendencia, no organizan las ideas de sus textos, ni saben cómo hacerlo, por lo que no siempre se puede advertir una secuencia lógica y diferencias entre la información principal y secundaria.

- No hacen acopio de ideas antes de escribir un texto, ni registran su pensamiento para después volcarlo en sus escritos.

- Ignoran la importancia que tienen los borradores de escritura para la calidad final de un texto.

- Desconocen las tipologías textuales y carecen de reglas que les ayuden a elaborar adecuadamente un texto.

- Desestiman el uso de trucos, reglas mnemónicas o recursos en la confección de un texto.

En sintonía con los resultados de este estudio y, teniendo en cuenta el modelo teórico que lo inspira, la enseñanza de estrategias se percibe como un procedimiento idóneo para 
contribuir al desarrollo de las habilidades cognitivas implicadas en la expresión escrita (Schunk, 2003; McCarthur, Graham y Fitzgerald, 2006; Monroe y Troia, 2006; Salvador, 2008; Gallego, García y Rodríguez, 2013, entre otros), se indican, a continuación, algunas actividades concretas para facilitar el aprendizaje de la planificación de la expresión escrita:

- Elaborar "torbellinos de ideas" para facilitar el acceso al conocimiento que se posee mediante la evocación de conceptos o ideas antes de escribir.

- Hacer listados de ideas que nos vienen a la mente pero sin desarrollar argumentos u ordenar las asociaciones.

- Manejar diccionarios de sinónimos y antónimos para incrementar la riqueza léxica del texto.

- Confeccionar borradores previos sobre temas de interés que, después, se utilicen en la redacción del texto.

- Pensar en los objetivos globales que se persiguen, es decir, es importante que desde el inicio quede claro cuál es la idea central que se pretende transmitir y argumentar y, a partir de ahí, establecer cómo se pueden hacer operativos esos objetivos.

- Confeccionar fichas o plantillas en las que se registren ideas o palabras clave relacionadas con el tema sobre el que se quiere escribir.

- Diferenciar las ideas principales y relacionarlas con las secundarias. Para desarrollar esta actividad se puede utilizar algún tipo de esquema, mapa conceptual o cuadro sinóptico que ayude a focalizar la cuestión a tratar.

- Elaborar fichas en las que se describan las reglas o principios de ordenación de los contenidos o partes que ha de tener un texto, atendiendo a su tipología (su estructura).

- Utilizar reglas mnemotécnicas de planificación guiada, cuya aplicación puede mejorar considerablemente la organización y estructuración del contenido.

- Concretar un plan para la construcción del texto escrito, lo que permite modelar distintas formas de representar las ideas (unir secuencias de dibujos o diagramas que muestren la historia en progresión, mapas conceptuales que revelen cómo evoluciona la historia en el tiempo y en el espacio, cronogramas que muestren una serie de eventos...).

Esta investigación, sin embargo, no está exenta de limitaciones, como por ejemplo, el localismo de los estudiantes con los que se ha trabajado o las propias peculiaridades de los estudios de caso que aconsejan cierta prudencia tanto a la hora de interpretar los datos como fundamentalmente a la hora de generalizarlos, de ahí que el estudio esté abierto a futuras 
comprobaciones o réplicas en las que se puede analizar el mismo proceso cognitivo u otros, manejar otros tipos de texto o contrastar los análisis de las declaraciones realizadas por los participantes de la investigación con los propios textos escritos (productos de escritura).

\section{Referencias}

Alamargot, Denis y Chanquoy, Lucile. (2001). Through the models of writing. Dordrecht: Kluwer Academic Press.

Alegría, Jesús y Carrillo, María Soledad. (2014). Mecanismos implicados en la escritura de palabras del castellano. Aula, (20), 45-64.

Aponte-Henao, Mónica y Zapata-Zabala, Maryoris Elena. (2013). Caracterización de las funciones cognitivas de un grupo de estudiantes con trastornos específicos del aprendizaje en un colegio de la ciudad de Cali, Colombia. Psychologia. Avances de la Disciplina, 7(1), 23-34.

Applebee, Arthur. (1981). Writing in secondary schools. Urbana IL: National Council of Teachers of English.

Bardin, Laurence. (1986). Análisis de contenido. Madrid: Akal.

Bayat, Nihat. (2014). The Effect of the Process Writing Approach on Writing Success and Anxiety. Educational Sciences: Theory \& Practice, 14(3), 1133-1141. DOI: 10.12738/estp.2014.3.1720.

Bereiter, Carl y Scardamalia, Marlene. (1987). The psychology of written composition. Hillsdale: Erlbaum.

Berninger, Virginia Wise y Whitaker, Denise. (1993). Theory Based Branching Diagnosis of Writing Disabilities. School Psychology Review, 22(4), 623-642.

Bosch, María y Fernández-Llebrez, Celia. (2014). Las pautas de lectura fácil como metodología de aproximación al trabajo en el aula: estudio de caso en estudiantes con síndrome de Down. Revista Síndrome de Down: Revista española de investigación e información sobre el Síndrome de Down, 31, 155-162.

Bruner, Jerome. (2004). Realidad mental y mundos posibles. Madrid: Alianza.

Calsamiglia, Helena. (2000). Estructura y funciones de la narración. Textos, 25, 9-21.

Camps, Anna. (1997). Escribir: la enseñanza y el aprendizaje de la composición escrita. Signos. Teoría y Práctica de la Educación, (20), 24-39.

Camps, Anna y Castelló, Monserrat. (1996) Las estrategias de enseñanza-aprendizaje en la escritura. En Carlos Monereo e Isabel Solé (Coords.), El asesoramiento psicopedagógico: una perspectiva profesional y constructivista (pp. 321-342). Madrid: Alianza. 
Canales, Ricardo C., Velarde, Esther M., Meléndez, Carmen M. y Lingán, Susana. (2013). Factores neuropsicológicos y procesos cognitivos en niños con retraso en la escritura y sin retraso en la escritura. Propósitos y Representaciones. Revista de Educación y Psicología de la USIL, 1(2), 11-29.

Castelló, Monserrat, González, Dolores e Iñesta, Ana. (2010). La regulación de la escritura académica en el doctorado: el impacto de la revisión colaborativa en los textos. Revista Española de Pedagogía, 68(247), 521-537.

Castelló, Monserrat y Monereo, Carlos. (1996). Un estudio empírico sobre la enseñanza y el aprendizaje de estrategias para la composición de textos argumentativos. Infancia y Aprendizaje, 74, 39-55.

Ceballos, Froilán Antonio. (2009). El informe de investigación con estudio de casos. Magis, Revista Internacional de Investigación en Educación, 1(2), 413-423.

Díaz-Itza, Eliseo y Miranda, Manuela. (2007). Perfiles gramaticales específicos en el síndrome de Down. Revista de Logopedia, Foniatría y Audiología, 27(4), 166-178.

Englert, Carol S. y Raphael, Taffy. (1988). Constructing well-formed prose: Process, structure and metacognitive knowledge. Exceptional Children, 54(6), 513-520.

Fox, David J. (1981). El proceso de investigación en educación. Pamplona: Eunsa.

Galeote, Miguel, Soto, Pilar, Sebastián, Eugenia, Rey, Rocío y Checa, Elena. (2012). La adquisición del vocabulario en niños con síndrome de Down: datos normativos y tendencias de desarrollo. Infancia y Aprendizaje, 35(1), 111-122. DOI: 10.1174/021037012798977502.

Gallego, José Luis. (2008a). La planificación de la expresión escrita por alumnos con retraso mental. Revista de Educación (Madrid), (346), 267-290.

Gallego, José Luis. (2008b). La planificación de la expresión escrita por alumnos de educación primaria. Bordón, 60(2), 63-76.

Gallego, José Luis y González, Joaquín. (2008). Cómo planifican la composición escrita alumnos con sobredotación intelectual. RIE. Revista de Investigación Educativa, 26(2), 463-484.

Gallego, José Luis y Rodríguez, Antonio. (2011). La planificación de la composición escrita por alumnado con discapacidad visual: un estudio de casos. Revista de Educación (Madrid), (356), 557-581. DOI: 10.4438/1988-592X-RE-2011-356-051.

Gallego, José Luis, García, Antonio y Rodríguez, Antonio. (2013). Cómo escriben los futuros docentes. Estrategias para la mejora. Málaga: Aljibe.

García, Antonio. (2009). Los procesos cognitivos y meta-cognitivos en la composición escrita del alumnado de etnia gitana con desventaja socioeducativa. Revista de Educación (Madrid), (348), 309-330. 
García, Antonio y Salvador, Francisco. (2010). Cómo escriben los alumnos de etnia gitana. Implicaciones para la praxis educativa. Madrid: EOS.

Gillham, Bill. (2000). Case study research methods. London: Continuum.

Gleason, Mary M., e Isaacson, Stephen. (2001). Using the new basals to teach the writing process: modification for students with learning problems. Reading and Writing Quaterly, 17(1), 75-92. DOI: 10.1080/105735601455747.

Gómez-Contreras, Flor Alejandra. (2011). La relevancia del error: hacia una didáctica de la autocorrección en escritos académicos. Revista de Educación y Desarrollo, (19), 13-20.

González, M., José y Martín, Isaías. (2006). Análisis del rendimiento en composición escrita y sus dificultades en Educación Secundaria. Infancia y Aprendizaje, 29(3), 315-326.

Graham, Steve. (2006). Strategy instruction and the teaching of writing. In Steve Graham, Charles A. MacArthur \& Jill Fitzgerald (Eds.), Handbook of writing research (pp. 187-207). New York: Guilford Press.

Graham, Steve y Harris, Karen. (2005). Improving the writing Performance of Young Struggling Writers: Theoretical and Programmatic Research From the Center on Accelerating Student Learning. The Journal of Special Education, 39(1), 19-33.

Guasch, Oriol y Ribas, Teresa. (2013). La entrevista en la investigación cualitativa sobre la didáctica de la lengua. Cultura y Educación, 25(4), 483-488, DOI: $10.1174 / 113564013808906852$.

Gutiérrez, Rafaela y Salvador, Francisco. (2006). El proceso de planificación en la expresión escrita de alumnos sordos: estudio de casos en Educación Secundaria. Revista de Educación (Madrid), (339), 435-453.

Hayes, John. (1996). A new framework for understanding cognition and affect in writing. In C. Michael Levy \& Sarah Ransdell (Eds.), The Science of Writing (pp. 1-27). Hillsdale, NJ: Lawrence Erlbaum Associates.

Hayes, John y Flower, Linda. (1980). Identifying the organization of writing process. In Lee W. Gregg \& Erwin R. Steinberg (Eds.), Cognitive process in writing (pp. 3-30). Hillsdale, NJ: Lawrence Erlbaum Associates.

Hayes, John y Nash, Jane. (1996). On the nature of planning in writing. In C. Michael Levy \& Sarah Ransdell (Eds.), The Science of Writing (pp. 29-55). Hillsdale: NJ, Lawrence Erlbaum Associates.

Hernández, Roberto, Fernández, Carlos y Baptista, Pilar. (2003). Metodología de la Investigación (3aㅡ ed.). México, D. F.: McGraw-Hill Interamericana.

Hidi, Suzanne y Boscolo, Pietro. (2006). Motivation and writing. In Charles A. MacArthur, Steve Graham \& Jill Fitzgerald (Eds.), Handbook of writing research (pp. 144-157). New York: Guilford Press. 
Hodder, lan. (2000). The interpretation of documents and material culture. In Norman K. Denzin \& Yvonna S. Lincoln (Eds.), Handbook of Qualitative Research (pp. 703-717). London: Sage Publications.

Jiménez, Alicia, Vicente, Florencio, Sánchez, Inmaculada, López, Manuel y Rodríguez, Marta. (2013). La evaluación morfosintáctica en adolescentes y jóvenes con Síndrome de Down. Campo Abierto, 32(2), 155-169.

Kellogg, Ronald T. (1994). The Psychology of writing. New York: Oxford University Press.

Krippendorff, Klaus. (2002). Metodología del análisis de contenido. Teoría y práctica. Barcelona: Paidós.

Lecuona, María del Pino; Rodríguez, María José y Sánchez, María Cruz. (2003). Evaluación de modelos de composición escrita en Educación Primaria. Revista de Educación (Madrid), (332), 301-326.

Lensmire, Timothy. (1994). When children write: critical re-visions of the writing workshop. New York: Teachers College Press.

Levy, C. Michael y Olive, Thierry. (2002). Real time studies in writing research: Progress and Prospects. In Thierry Olive \& C. Michael Levy (Eds.), Contemporary tools and techniques for studying writing (pp. 1-8). Dordrecht, A.A.: Kluwer Academic Publishers.

Marinkovich, Juana. (2002). Enfoques de proceso en la producción de textos escritos. Revista Signos, 35(51-52), 217-230.

McCarthur, Charles A., Graham, Steve y Fitzgerald, Jill. (2006). Handbook of writing research. New York: Guilford Press.

McCutchen, Deborah. (1995). Cognitive processes in children's writing: Developmental and individual differences. Issues in Education: Contributions from Educational Psychology, 1, 123-160.

McCutchen, Deborah. (2006). Cognitive factors in the development of children`s writing. In Charles A. McCarthur, Steve Graham \& Jill Fitzgerald (Eds.), Handbook of writing research (pp. 115-130). New York: Guilford Press.

Miles, Matthew B. y Huberman, A. Michael. (1994). Qualitative Data Analysis: An Expanded Sourcebook ( $2^{\mathrm{a}}$ ed.). Thousand Oaks, California: Sage Publications.

Monroe, Brandon W. Y Troia, Gary. (2006). Teaching Writing Strategies to Middle School Students with Disabilities. The Journal of Educational Research, 100(1), 21-33.

Newcomer, Phyllis L. y Barenbaum, Edna M. (1991). The written composition ability of children with learning disabilities: A review of the literature from 1980 to 1990. Journal of Learning Disabilities, 24(10), 578-593. 
Ochoa, Solanlly y Aragón, Lucero. (2007). Funcionamiento metacognitivo de estudiantes universitarios durante la escritura de reseñas analíticas. Universitas Psychologica, 6(3), 493-506.

Pereira, Cecilia y di Stefano, Mariana. (2007). El taller de escritura en posgrado: Representaciones sociales e interacción entre pares. Signos, 40(64), 405-430.

Pérez, María Nélida. (2010). Elecciones lingüísticas en el proceso de planificación de un ensayo. Revista Signos, 43(72), 125-152.

Ramos, José Luis; Cuadrado, Isabel e Iglesias, Beatriz. (2005). La composición escrita en el alumnado de Educación Primaria y Secundaria. Cultura y Educación, 17(3), 239-251.

Re, Anna Maria, Pedron, Martina y Cornoldi, Cesare (2007). Expressive writing difficulties in children described as exhibiting ADHD symptoms. Journal of Learning Disabilities, 40(3), 244-255.

Robles, María Auxiliadora. (2010). Respuesta a la lectura de un grupo de niños con síndrome de Down de un programa de Atención Temprana. Apuntes de Psicología, 28(3), 367376.

Rodríguez, Antonio. (2007). Disfunciones sintácticas encontradas en textos narrativos producidos por alumnos con baja visión e invidencia. Revista de Educación (Madrid), (343), 531-451.

Rodríguez-Parra, María J., Buiza, Juan J., Adrián, José A. y Alegría, Jesús. (2012). Lengua oral, bilingüismo y dificultades en el aprendizaje de la lengua escrita. Infancia y Aprendizaje, 35(3), 343-364. DOI: 10.1174/021037012802238966.

Ruiz, Uri, y Camps, Anna. (2009). Investigar los géneros discursivos en el proceso educativo. Revista de Psicodidáctica, 14(2), 211-228

Sabaj, Omar. (2009). Descubriendo algunos problemas en la redacción de Artículos de Investigación Científica (AIC) de alumnos de postgrado. Revista Signos, 42(69), 107127.

Salvador, Francisco (Ed.). (2005). La expresión escrita de alumnos con necesidades educativas especiales. Procesos cognitivos. Málaga: Aljibe.

Salvador, Francisco. (2008). Psicopedagogía de la lengua escrita. Madrid: EOS.

Salvador, Francisco y García, Antonio. (2009). El proceso de revisión en la composición escrita de alumnos de educación primaria. Revista Española de Pedagogía, 67(242), 61-76.

Scardamalia, Marlene y Bereiter, Carl. (1986). Research on written composition. In Merlin C. Wittrock (Ed.), Handbook of research on teaching. (3ª ed, pp. 778-803). Nueva York: McMillan.

Scardamalia, Marlene y Bereiter, Carl. (1992). Dos modelos explicativos de los procesos de composición escrita. Infancia y Aprendizaje, 58, 43-60. 
Schunk, Dale H. (2003). Self-efficacy for reading and writing influence of modeling self, goal setting, and self-evaluation. Reading and Writing Quarterly, 19, 159-172.

Sperling, Melanie y Freedman, Sarah Warshauer. (2001). Research on writing. In Virginia Richardson (Ed.), Handbook of research on teaching (pp. 370-389). Washington: American Educational Research Association.

Stake, Robert. (2010). Investigación con estudio de caso. Madrid: Morata.

Tolchinsky, Liliana, Ribera Paulina y García-Parejo, Isabel. (2012). Conocimientos iniciales y logros de los alumnos en el aprendizaje de la lectura y la escritura. Cultura y Educación, 24(4), 415-433. DOI: 10.1174/113564012803998811.

Torrance, Mark, Thomas, Glyn V. \& Robinson, Elizabeth J. (2000). Individual differences in undergraduate essay-writing strategies: a longitudinal study. Higher Education, 39(2), 181-200.

Troia, Gary. (2006). Writing instruction for students with learning disabilities. In Charles A. MacArthur, Steve Graham \& Jill Fitzgerald (Eds.), Handbook of writing research (pp. 324336). New Cork: Guilford Press.

Troncoso, M., Victoria y Flórez, Jesús. (2011). Comprensión en la lectura de las personas con síndrome de Down. Revista Síndrome de Down: Revista española de investigación e información sobre el Síndrome de Down, 28, 50-59.

Velarde, Esther y Canales, Ricardo. (2014). Procesos psicológicos de la escritura y diferencias neuropsicológicos y cognitivas según nivel socioeconómico y dominio escritor en estudiantes de la provincia constitucional del Callao-Perú. Estudios de Psicología, 35(3), 584-604, DOI: 10.1080/02109395.2014.965461.

Vygotsky, Lev S. (1986). Thought and language. Cambridge, MA: MIT Press.

Williams, Joanna P. (2003). Teaching text structure to improve reading comprehension. In Lee Swanson, Karen Harris y Steve Graham (Eds.), Handbook of learning disabilities (pp. 293-305). New York: Guilford Press.

Yin, Robert K. (2003). Case study research: Design and methods (3 ed.). Thousand Oaks, CA: Sage. 